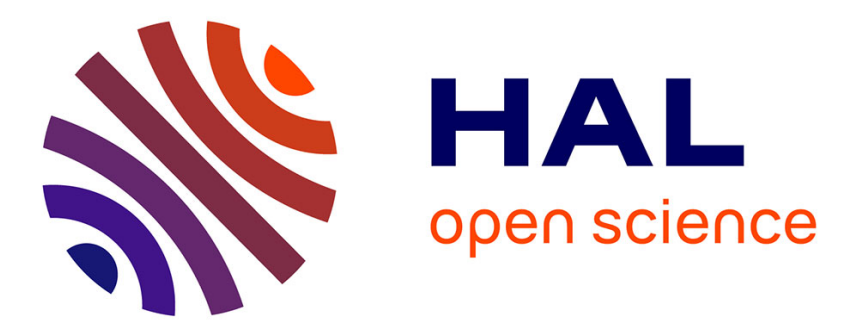

\title{
Application of the Asymptotic Numerical Method to the Coanda study
}

Cyrille Allery, Jean-Marc Cadou, Aziz Hamdouni, Dina Razafindralandy

\section{To cite this version:}

Cyrille Allery, Jean-Marc Cadou, Aziz Hamdouni, Dina Razafindralandy. Application of the Asymptotic Numerical Method to the Coanda study. Revue Européenne des Éléments Finis, 2004, 13 (1-2), pp.57-77. 10.3166/reef.13.57-77 . hal-00312148

\section{HAL Id: hal-00312148 \\ https://hal.science/hal-00312148}

Submitted on 22 Jun 2018

HAL is a multi-disciplinary open access archive for the deposit and dissemination of scientific research documents, whether they are published or not. The documents may come from teaching and research institutions in France or abroad, or from public or private research centers.
L'archive ouverte pluridisciplinaire HAL, est destinée au dépôt et à la diffusion de documents scientifiques de niveau recherche, publiés ou non, émanant des établissements d'enseignement et de recherche français ou étrangers, des laboratoires publics ou privés. 


\title{
Application of the Asymptotic Numerical Method to the Coanda effect study
}

\section{Cyrille Allery* $^{*}$ Jean-Marc Cadou** ${ }^{* *}$ Aziz Hamdouni* Dina Razafindralandy*}

\author{
* LEPTAB, Université de La Rochelle \\ Avenue Michel Crépeau \\ 17042 La Rochelle Cédex 1, France \\ callery@univ-lr.fr,ahamdoun@univ-lr.fretdrazafin@univ-lr.fr \\ ${ }^{* *}$ Laboratoire Génie Mécanique et Matériaux \\ Rue de Saint Maudé, BP 92116 \\ 56321 Lorient Cédex, France \\ jean-marc.cadou@iuplo.univ-ubs.fr
}

ABSTRACT. The phenomenon of jet attachment to a wall by Coanda effect is very important in many industrial applications (sound reduction, bad dispersion of the air in a ventilated room...). In order to control it, it is necessary to know its occurrence according to the various parameters of the flow. In this article the asymptotic numerical method (ANM) is used to study the Coanda effect which occurs in several flow configurations (sudden expansion with or without divergent and open cavity). The ANM is a fast, efficient and robust method to determine these stationary bifurcations. The attachement Reynolds numbers obtained with the ANM are compared to the results found in the literature. Finally, evolution laws of critical Reynolds numbers according to the various geometrical parameters are proposed.

RÉSUMÉ. Le phénomène d'attachement d'un jet à une paroi par effet Coanda, qui se retrouve dans de nombreuses applications industrielles, joue un rôle important (réduction sonore, mauvaise dispersion de l'air dans une pièce ventilée ...). Afin de le contrôler, il est nécessaire de connaître son apparition en fonction des différents paramètres caractérisant l'écoulement. Dans cet article la méthode asymptotique numérique (MAN) est utilisée pour étudier l'effet Coanda qui se produit dans différentes configurations d'écoulement (expansion brusque avec ou sans divergent et cavité ouverte). La MAN s'avère être un moyen efficace, robuste et très rapide pour déterminer ces bifurcations stationnaires. Ainsi après avoir comparé les nombres de Reynolds d'attachement obtenus par la MAN à ceux existant dans la littérature, des lois d'évolution du nombre de Reynolds critique en fonction des différents paramètres géométriques sont proposées. KEYWORDS: ANM, stationary bifurcation, Coanda effect, sudden expansion, open cavity, diffuser. MOTS-CLÉS : MAN, bifurcation stationnaire, Effet Coanda, Expansion brusque, cavité ouverte. 


\section{Introduction}

It is well known that when a body is immersed in a flow, the jet shows a strong tendency to become attached to the solid surface. This phenomenon of attachment, called Coanda effect, is intensively used in industrial applications to control the flows. In particular, this effect was applied, by Coanda, to improve the scavenging of internal combustion engines, to produce nozzle with high thrust augmentation and to increase the maximum lift coefficient of a wing. It is also the origin of an important noise reduction in the air flow crossing two diaphragms in tandem inside a cylindrical duct [GUE 01]. In this case, the Coanda effect, which is associated to an hysteresis loop, appears by the attachment of a jet to the diffuser walls. Therefore, to obtain the maximum noise reduction, it is necessary to control the Coanda effect. The jet attachment is also used in ventilation to stabilize the injected jet and to prevent that, under the gravity effect, it does not fall too quickly into the room. In this case, the Coanda effect can also be avoided. Indeed, for an air-conditioned room, the Coanda effect can deteriorate the mixing of the injected air with the air of the room.

Thus to be able to control the advantages or the drawbacks related to the Coanda effect, it is necessary to know its dependency with respect to the various parameters of the problem (the Reynolds number or the geometrical parameters). For these reasons the numerical asymptotic method (ANM) is used in the work. Actually, this technique, unlike the usual methods used in fluid mechanics, allows to compute the totally solution paths, that is very well adapted to a parametric study.

Usually, the nonlinear equations depending on a single parameter, like the equations governing the viscous fluid flow, are solved by using prediction-correction methods, the most used being the incremental iterative Newton-Raphson method. These methods have been successfully applied to a wide variety of nonlinear problems, but do not take advantage of the special nature of the given non-linearity. Therefore the computing time is generally large. When the non linearity is quadratic, a possible alternative, is the use of an Asymptotic Numerical Method (ANM). Let us note that when non linearity is more than quadratic, ANM can also be used, see [POT 97] for more explanations. This method, based on the coupling between a technique of perturbation and a finite element discretization ${ }^{1}$ allows to transform the initial non linear problem into a series of linear problems. Those discretizated problems have the same matrix of rigidity which is assembled and triangulated only once. Consequently, the ANM is very efficient and much less expensive than standard methods [CAD 97]. Associated to a continuation method [COC 94], the ANM becomes an automatic and efficient algorithm to obtain solution paths of a non-linear problem.

The asymptotic numerical methods were suggested in solid mechanics in 1968 by Thompson and Walker [THO 68] and, in fluid mechanics in 1976 by Kawahara et al. [KAW 76]. But they were intensively used only at the end of the eighties, due to the progress in computing power and in numerical techniques. Then, these methods have

1. It is possible to use a finite volume discretization [ALL 02]. 
been successfully applied in various fields of solid mechanics, in static (rod, plate and shell problems) or in dynamic conditions (non linear vibrations). In the middle of years 90, the ANM was applied to the resolution of the Navier-Stokes equations (Hadji [HAD 97], Tri [TRI 96], Cadou [CAD 97], Cadou et al. [CAD 01]). Associated to a continuation method, the ANM leads to compute the non linear fundamental branch. It is also proved to be an effective way for the resolution of bifurcated paths. Indeed close to a bifurcation point, the ANM gives an accumulation of steps. A modification of ANM parameters leads to compute bifurcated branches. In the fluid mechanics scope, Tri et al. [TRI 96] has associated the ANM to a stationary bifurcation indicator which has been initially proposed by Boutyour [BOU 94]. Moreover bifurcating branches can also be computed, see Vannuci et al. [VAN 98]. First, they introduce a perturbation in the ANM equations, and then perform a linearization.

In this paper, we describe the bases of Asymptotic Numerical Method in Fluid Mechanics. This technique is then applied to characterize the Reynolds number of attachment of a jet to a wall in the case of : a sudden expansion configuration with or without divergent and in the open cavity configuration. The influence of geometrical parameters on the critical Reynolds numbers is determined. The ANM results allow us to define evolution laws of the critical Reynolds number according to the other parameters.

\section{Asymptotic Numerical Method (ANM)}

\subsection{Position of the problem}

We consider a bounded domain $\Omega$ of $\mathbb{R}^{2}$; the boundary is noted $\Gamma$. In this domain, we consider a Newtonian and incompressible fluid. The fluid density is denoted $\rho$ and the dynamic viscosity $\mu$. In each position $x$ of $\Omega$ and for each time $t$, the velocity field is written $u(x, t)$ and the pressure is noted $p(x, t)$. The boundary $\Gamma$ is divided into $\Gamma=\Gamma_{u} \cup \Gamma_{\sigma}$. Boundary conditions of Dirichlet type are imposed on $\Gamma_{u}$ and boundary conditions of Neumann type are imposed on $\Gamma_{\sigma}$. The body forces are neglected. The governing equations are :

$$
\operatorname{div}(u)=0 \quad \text { and } \quad \frac{\partial u}{\partial t}+\operatorname{Div}(u \otimes u)=\operatorname{Div}(\sigma)
$$

with the following boundary and initial conditions :

$$
u(x, t)=f(x, t) \text { on } \Gamma_{u} \quad \sigma n=h(x, t) \text { on } \Gamma_{\sigma} \quad \text { and } \quad u\left(x, t_{0}\right)=g(x)
$$

$n$ is the outward normal to $\Gamma_{\sigma}$ and $\sigma$ is the stress tensor defined by $\sigma=-p I+\tau$. The viscous stress tensor $\tau$ is defined by $\tau=\mu\left(\operatorname{gradu}+{ }^{t} \operatorname{gradu}\right)$ where ${ }^{t}(\bullet)$ corresponds to the transpose operator.

The resolution of Navier-Stokes equations (1) with the incompressibility condition, requires the computation of consistent pressure and velocity field which must 
satisfy the motion equations and the mass conservation equation. This is difficult to negotiate because the pressure does not explicitly appear in the mass conservation equation. To solve this problem several methods are possible : prediction-correction algorithms, projection methods or a penalization method. In this work the latter technique is used. Even if this technique is less accurate than the other ones, it has the advantage to keep the efficiency of the asymptotic numerical method. With the penalization technique the incompressibility condition is :

$$
\operatorname{divu}-\frac{p}{\kappa}=0
$$

where $\kappa$ is a very large parameter.

\subsection{Variational formulation of Navier-stokes equations}

The previous problem (1) can be written into variational form. $L(U)$ and $Q(U, U)$ are linear and quadratic operators of the unknown mixed vector $U(U=(u, p))$. The boundary conditions of Dirichlet type are taken in the following form $u=\lambda u_{d}$ where $\lambda$ is a parameter which can be identified to the Reynolds number. We obtain thus :

Find $U=\left\{\begin{array}{l}u \\ p\end{array}\right\}$ solution of $\left\{\begin{array}{l}\frac{d}{d t} M(U)+L(U)+Q(U, U)=0 \text { in } \Omega \\ u=\lambda u_{d} \text { on } \Gamma_{u}\end{array}\right.$

$$
\text { with }\left\{\begin{array}{c}
(M(U), \delta U)=\rho \int_{\Omega}{ }^{t} \delta u u d x \quad(Q(U, U), \delta U)=\rho \int_{\Omega}{ }^{t} \delta u D i v(u \otimes u) d x \\
(L(U), \delta U)=-\int_{\Omega} p d i v \delta u d x+\int_{\Omega} \operatorname{Tr}\left(\tau \frac{\partial \delta u}{\partial x}\right) d x \\
-\int_{\Omega} \delta p \operatorname{divudx}+\frac{1}{\kappa} \int_{\Omega} p \delta p d x-\int_{\Gamma_{\sigma}}{ }^{t} \delta u h d \Gamma
\end{array}\right.
$$

for all $\delta u \in V=\left\{\delta u \in\left\{H^{1}(\Omega)\right\}^{2}\right.$ such as $\left.\left.\delta u\right|_{\Gamma_{u}}=0\right\}$ and all $\delta p \in L^{2}(\Omega)$.

We consider now only the stationary case (i.e $\frac{d}{d t} M(U)=0$ ) and homogeneous Neumann boundary conditions (i.e $h=0$ ).

\subsection{Perturbation method}

The ANM is based on a perturbation technique. In this way, from a regular and solution point $\left(U_{0}, \lambda_{0}\right)$ we expand the unknowns $(U, \lambda)$ in series with respect to a path parameter " $a$ " :

$$
U=U_{0}+a U_{1}+a^{2} U_{2}+\ldots \quad \text { and } \quad \lambda=\lambda_{0}+a \lambda_{1}+a^{2} \lambda_{2}+\ldots
$$


For a well posed problem, the parameter " $a$ "must be defined. We simply define $\mathrm{it}^{2}$ with the projection of velocity increment $u-u_{0}$ and parameter increment $\lambda-\lambda_{0}$ on the tangent vector $\left(u_{1}, \lambda_{1}\right)$ :

$$
\left.a=\frac{1}{s^{2}}\left\{\left(u-u_{0}, u_{1}\right)+\left(\lambda-\lambda_{0}\right) \lambda_{1}\right)\right\}
$$

where $(\bullet, \bullet)$ is the inner product in $L^{2}(\Omega)$ and $s$ is a scalar.

The developments (3) are then introduced into (2). Equating powers of " $a$ ", we obtain a succession of linear problems :

$$
\begin{array}{ll}
\text { Order 1: } & \text { Order } q \geq 2: \\
\begin{cases}L_{t}\left(U_{1}\right)=0 \\
u_{1}=\lambda_{1} u_{d} \text { on } \Gamma_{u} \\
\left(u_{1}, u_{1}\right)+\lambda_{1} \lambda_{1}=s^{2}\end{cases} & \left\{\begin{array}{l}
L_{t}\left(U_{q}\right)=-\sum_{r=1}^{q-1} Q\left(U_{r}, U_{q-r}\right) \\
u_{q}=\lambda_{q} u_{d} \text { on } \Gamma_{u} \\
\left(u_{q}, u_{1}\right)+\lambda_{q} \lambda_{1}=0
\end{array}\right.
\end{array}
$$

where $L_{t}$ is the tangential operator defined by :

$$
L_{t}(\bullet)=L(\bullet)+Q\left(U_{0}, \bullet\right)+Q\left(\bullet, U_{0}\right)
$$

The ANM allows therefore to reduce the Navier-Stokes equations (1), which are non linear, to a serie of linear problems which have the same tangential operator $L_{t}$. Then, only one inversion or decomposition of matrix is needed to solve (5). The second member is built with the solutions computed at the previous orders.

\subsection{Discretization by a Galerkin formulation-Condensation}

\section{a) Discretization}

The finite elements used are quadrangles with 9 velocity nodes and 3 for pressure. Thus, the pressure interpolation functions are linear and those of the velocity field are quadratic. The pressure is then discontinuous between the elements. This leads, with the penalization of the incompressibility equation, to build a weak numerical scheme in pressure. For a Galerkin formulation, the unknowns $(u, p)$ and the test functions $(\delta u, \delta p)$ are approximated by :

$$
\{u\}=\left[N_{u}\right]\{\bar{u}\} \quad\{\delta u\}=\left[N_{u}\right]\{\delta \bar{u}\} \quad \text { and } \quad p={ }^{t}\left\{N_{p}\right\}\{\bar{p}\} \quad \delta p={ }^{t}\left\{N_{p}\right\}\{\delta \bar{p}\}
$$

2. Cochelin [COC 94] suggests other definitions of this parameter. 
where $\left[N_{u}\right]$ (resp. $\left\{N_{p}\right\}$ ) are the velocity (resp. pressure) shape functions and $\{\bullet\}$ are the nodal unknown factors. By introducing these definitions in the system of equations (5), the discrete problem at the order $q$ is written :

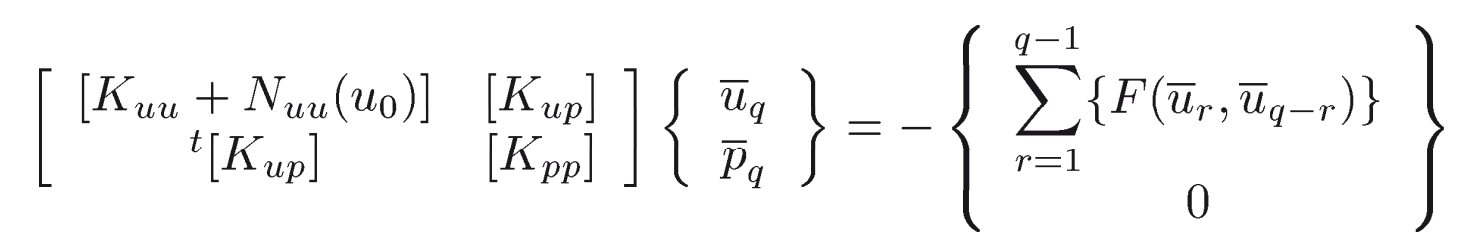

$$
\begin{aligned}
& \int\left[K_{u u}\right]=\sum_{n e l} \int_{\Omega_{e}}{ }^{t} B C B d x \quad\left[K_{u p}\right]=-\sum_{n e l} \int_{\Omega_{e}}{ }^{t} B_{v}{ }^{t} N_{p} d x \\
& \text { with }\left\{\begin{array}{l}
{\left[N_{u u}\left(\bar{u}_{0}\right)\right]=\rho \sum_{n e l} \int_{\Omega_{e}}{ }^{t} N_{u}\left\{u_{0} \operatorname{Grad}\left(N_{u}\right)+\operatorname{Grad}\left(u_{0}\right) N_{u}\right\} d x} \\
{\left[K_{p p}\right]=\frac{1}{\kappa} \sum_{n e l} \int_{\Omega_{e}} N_{p}{ }^{t} N_{p} d x \quad F\left(\bar{u}_{r}, \bar{u}_{q-r}\right)=\rho \sum_{n e l} \int_{\Omega_{e}}{ }^{t} N_{u} u_{r} \operatorname{Grad}\left(u_{q-r}\right) d x}
\end{array}\right.
\end{aligned}
$$

The matrix $B$ connects the deformations $D$ to the nodal field of velocity, i.e. ${ }^{3}\{D\}=$ $B\{\bar{u}\}$. It contains the derivatives of the velocity shape functions. $C$ is a $3 \times 3$ matrix which allows to write the behavior law between ${ }^{4}\{\tau\}$ and $\{D\}$. The line vector $B_{v}$ allows to connect the divergence of velocity to the nodal unknown factors, so $\operatorname{divu}=B_{v}\{\bar{u}\}$.

The discrete form of the path parameter is :

$$
{ }^{t}\left\{\bar{u}_{q}\right\}\left\{\bar{u}_{1}\right\}+\lambda_{q} \lambda_{1}=0
$$

\section{b) Condensation of the pressure}

The condensation method, which consists in eliminating the unknown factors of nodal pressure $\{\bar{p}\}$, is done at the elementary level. The matrix $K_{p p}$ being invertible the pressure at the order $q$ is defined by

$$
\left\{\bar{p}_{q}\right\}=-\left[K_{p p}\right]^{-1 t}\left[K_{u p}\right]\left\{\bar{u}_{q}\right\}
$$

By introducing this relation into the discrete equations of momentum (6), the problem to be solved is :

$$
\left[K_{t}\left(\bar{u}_{0}\right)\right]\left\{\bar{u}_{q}\right\}=-\sum_{r=1}^{q-1}\left\{F\left(\bar{u}_{r}, \bar{u}_{q-r}\right)\right\}
$$

where $\left[K_{t}\left(\bar{u}_{0}\right)\right]$ is the tangential matrix defined by

$$
\left[K_{t}\left(\bar{u}_{0}\right)\right]=\left[K_{u u}+N_{u u}\left(\bar{u}_{0}\right)\right]-\left[K_{u p}\right]\left[K_{p p}\right]^{-1 t}\left[K_{u p}\right]
$$

3. $\{D\}={ }^{t}\left(D_{11}, D_{22}, 2 D_{12}\right)$ with $D=\frac{1}{2}\left(G r a d u+{ }^{t}\right.$ Gradu $)$.

4. $\{\tau\}={ }^{t}\left(\tau_{11}, \tau_{22}, \tau_{12}\right)$ 
The resolution of the previous system (9) gives the values of the nodal velocities $\bar{u}_{q}$ for each increment $\lambda$ (or for each value of Reynolds number). The pressure is obtained by introducing the computed velocity $\bar{u}_{q}$ into (8).

\section{c) Taking into account applied velocities}

The applied velocities $u=\lambda u_{d}$ on $\Gamma_{u}$ are modified into given loads on the edge [BAT 90]. The equation (9) becomes

$$
\left[K_{t}\left(\bar{u}_{0}\right)\right]\left\{\bar{u}_{q}\right\}=\lambda_{q}\left\{F_{u}\right\}-\sum_{r=1}^{q-1}\left\{F\left(\bar{u}_{r}, \bar{u}_{q-r}\right)\right\}
$$

where $\left[K_{t}\left(\bar{u}_{0}\right)\right]$ is the tangential matrix, after taking into account the imposed velocities, and $F_{u}$ is the applied load.

Finally the system of discrete linear problems to be solved is written :

\section{Order 1 :}

$$
\left\{\begin{array}{l}
{\left[K_{t}\left(\bar{u}_{0}\right)\right]\left\{\bar{u}_{1}\right\}=\lambda_{1}\left\{F_{u}\right\}} \\
{ }^{t}\left\{\bar{u}_{1}\right\}\left\{\bar{u}_{1}\right\}+\lambda_{1}^{2}=s^{2}
\end{array}\right.
$$

\section{Order $q \geq 2$ :}

$$
\left\{\begin{array}{l}
{\left[K_{t}\left(\bar{u}_{0}\right)\right]\left\{\bar{u}_{q}\right\}=\lambda_{q}\left\{F_{u}\right\}-\sum_{r=1}^{q-1}\left\{F\left(\bar{u}_{r}, \bar{u}_{q-r}\right)\right\}} \\
{ }^{t}\left\{\bar{u}_{q}\right\}\left\{\bar{u}_{1}\right\}+\lambda_{q} \lambda_{1}=0
\end{array}\right.
$$

The scalar $s$ which appears at order 1 is a parameter imposed by the user (usually equal to 1$)$ and the initial field $\left(U_{0}, \lambda_{0}\right)$ is solution of (2). The resolution of the previous problem (12) leads to the solution in the following integro-power series of $a$ :

$$
U=U_{0}+a U_{1}+a^{2} U_{2}+a^{3} U_{3}+\ldots
$$

\subsection{Method of continuation}

As the convergence radius of series (13) is finite, the solutions computed with ANM are only valid up to a certain value of the path parameter ${ }^{5} a$. To compute the whole solution branch, a continuation method is used. This technique, suggested initially by Cochelin[COC 94], consists in introducing into the equations a new starting point $\left(U_{0}, \lambda_{0}\right)$, located inside the range of validity of the series. By applying once again the ANM from this point, an additional part of the curve solution is obtained. By using this technique successively, we obtain the whole curve solution. The only difficulty lies on the evaluation of a new starting point $\left(U_{0}, \lambda_{0}\right)$. Cochelin has suggested a simple criterion ${ }^{6}$ coming from the fact that two successive polynomial approximations move away brutally as soon as the radius of convergence is reached. Thus, this criterion

5. So of the Reynolds number because $R e=g\left(\lambda, u_{\text {ref }}, d_{r e f}\right)$ and $a=f(u, \lambda)$.

6 . A second criterion based on the residual method exists. 
(criterion of velocity), which takes a very small computing time, consists in requiring that the difference between two consecutive orders of the series is lower than a given parameter $\varepsilon$. That results in :

$$
\frac{\left\|S_{n}-S_{n-1}\right\|}{\left\|S_{n}-S_{0}\right\|}=\frac{\left\|a^{n} u_{n}\right\|}{\left\|a u_{1}+\ldots+u_{n}\right\|}<\varepsilon
$$

with $S_{n}=\sum_{k=1}^{n} a^{k} u_{k}$ is a polynomial approximation. The continuation method is then automatic. The step length is adjusted according to the difficulty encountered on the curve solution.

\subsection{Indicator of stationary bifurcation}

Tri et al. [TRI 96], Cadou [CAD 97] have introduced an indicator of stationary bifurcation for the incompressible fluid flows. This indicator, which is well adapted to the polynomial approximations previously described, is explained in this section.

Let us suppose that the path $\left(U_{f}, \lambda_{f}\right)$ which passes through a given point $\left(U_{0}, \lambda_{0}\right)$ solution of the problem (12) is known. To detect, on this fundamental path $\left(U_{f}, \lambda_{f}\right)$, the possible stationary bifurcation in the vicinity of $\left(U_{0}, \lambda_{0}\right)$, we introduce a disturbance load $\beta \mathcal{F}$ where $\beta$ represents the intensity of the load and $\mathcal{F}$ is a random load vector. The response to this disturbance is a fluctuation of velocity $\Delta \nu$ such as $U=U_{f}+\Delta \nu$.

This relation is introduced into the Navier-Stokes equations (2). By neglecting the non linear terms in $\Delta \nu$, the disturbed equilibrium problem is written :

$$
\begin{cases}L_{t}(\Delta \nu)=\beta \mathcal{F} & \text { in } \Omega \\ \Delta \nu=0 & \text { on } \Gamma_{u} \\ \left(\Delta \nu-\Delta \nu_{0}, \Delta \nu_{0}\right)=0 & \end{cases}
$$

where $L_{t}(\Delta \nu)$ is the tangential operator defined by

$$
L_{t}(\Delta \nu)=L(\Delta \nu)+Q\left(U_{f}, \Delta \nu\right)+Q\left(\Delta \nu, U_{f}\right)
$$

An orthogonality condition $\left(\Delta \nu-\Delta \nu_{0}, \Delta \nu_{0}\right)=0$ is added in order to have a well posed problem. The disturbance intensity $\beta$ is associated to a measurement of rigidity. A stationary bifurcation point on the fundamental path, corresponds to the loss of this rigidity $(\beta=0)$. Thus, the scalar $\beta$ defined along the path is equal to zero in all points where the operator $L_{t}$ is singular (bifurcation or limit point). Therefore, it is the indicator of bifurcation.

After discretization by finite elements of the problem (15) the indicator $\beta$ and the bifurcation mode $\Delta \nu$ are written :

$$
\beta=\frac{<\Delta \nu_{0}, \Delta \nu_{0}>}{<\left[K_{t}\left(U_{f}\right)\right]^{-1}\{\mathcal{F}\}, \Delta \nu_{0}>} \quad \text { and } \quad\{\Delta \nu\}=\beta\left[K_{t}\left(U_{f}\right)\right]^{-1}\{\mathcal{F}\}
$$


where $\left\{\Delta \nu_{0}\right\}$ is solution of $\left\{\Delta \nu_{0}\right\}=\left[K_{t}\left(U_{0}\right)\right]^{-1}\{\mathcal{F}\}$

In the numerical simulations, at each point of the fundamental path, the bifurcation indicator is obtained by (16). Finally, the numerical experiments [CAD 97] have shown that in the vicinity of a bifurcation (as well as limit points) a phenomenon of step accumulation occurs. So it is not necessary to compute the indicator on the whole fundamental path, but only where these zones of step accumulations appear. It is a visual criterion of detection of bifurcation which is refined by the resolution of the equations (16).

The technique, reported in this section, will be applied to detect the stationary bifurcation phenomenon due to the Coanda effect. The numerical scheme features (truncation order, parameter $\varepsilon$, mesh) will be given during the study of each flow configuration.

\section{Application of the ANM to the Coanda effect}

In this section, the ANM is applied to study the evolution of the attachment Reynolds number of a jet to a wall versus the various geometrical parameters. Three particular two-dimensional configurations are considered and presented on figure 1 : sudden expansion with or without divergent and open cavity. The air flow through these three configurations is considered. The Reynolds number is given by $R e=\frac{U_{0} h}{\nu}$ where $U_{0}$ is the maximum velocity at the inlet of the jet, $h$ is the inlet height and $\nu$ is the kinematic viscosity of the fluid. At the inlet of the channel a parabolic velocity profile is imposed. Non-slip boundary conditions $(u=0)$ are applied along the rigid walls. At the exit, the boundary condition $\sigma . n=0$ is applied where $\sigma$ is the stress tensor and $n$ the outward normal of exit border $\Gamma_{\sigma}$.

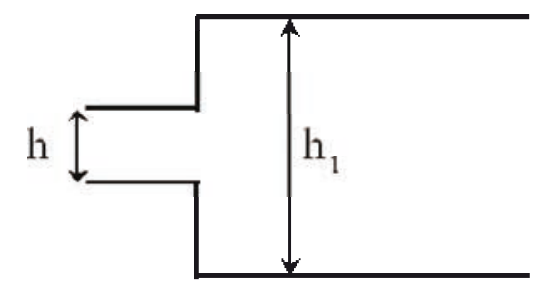

a) sudden expansion

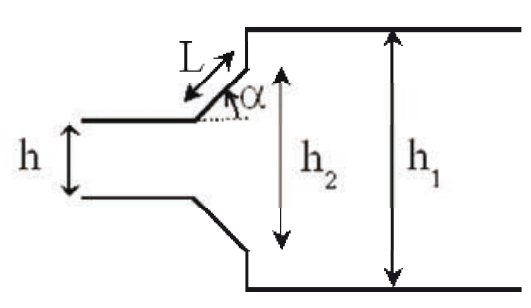

b) sudden expansion with divergent

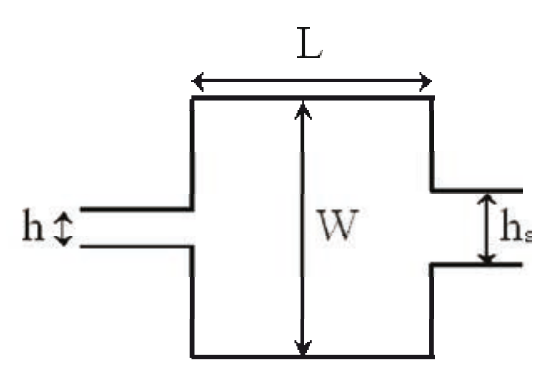

c) open cavity

Figure 1. Geometry studied in the article. A basis $(O x, y)$, whose origin $O$ is at middle height of the exit of the inlet, is introduced

The stationary bifurcation, which is characterized by the loss of the flow symmetry, may be obtained with the ANM in two ways : either by using the indicator of bifurcation presented in the previous section, or by following the evolution of the ver- 
tical velocity $v$ in a point on the axis of symmetry (Oy) versus the Reynolds number. In this last case the bifurcation Reynolds number corresponds to the one for which $v$ becomes equal to zero. These two ways were applied to the study of the flow through a sudden expansion. We have noted that the maximum difference concerning the prediction of the bifurcation Reynolds number was very small (around 1\%). Thereafter, the critical Reynolds number is the Reynolds number for which the vertical velocity in a point of the axis is equal to 0.01 time the highest velocity.

\subsection{Study of a sudden expansion}

For expansion ratios ( $E=h_{1} / h$ where $h$ is the height of the inlet channel and $h_{1}$ the height of the exit channel) higher than 1.5 and for low Reynolds numbers, the flow into a sudden expansion is symmetrical. It is characterized by the presence of two equal zones of recirculation located on both sides of the central axis. When the velocity increases, a stationary instability appears, which breaks the symmetry of the flow. One zone of recirculation increases more quickly than the other, which implies the attachment of the jet to one of the expansion walls. This behavior ${ }^{7}$ is represented on figure 2 for a sudden expansion $(E=2)$. This phenomenon of attachment is attributed to the Coanda effect and is interpreted in the following way : under the impulse of a disturbance, the jet is deflected towards one of the walls leading to a velocity increasing along this wall and, thus, to a pressure decreasing. This pressure difference between the two walls accentuates the deflection of the jet and involves its attachment to the wall. For low Reynolds numbers, there is a single symmetrical stable path. When Reynolds number is high, a supercritical bifurcation occurs where two asymmetrical stable states can exist [MIZ 00]. For larger Reynolds numbers, the flow oscillates periodically in time and this characterizes a Hopf bifurcation. On the other hand, if the expansion ratio $E$ is small, Abott and Kline [ABB 62] showed that a Hopf bifurcation exists and that the flow oscillates without passing through a stationary bifurcation.

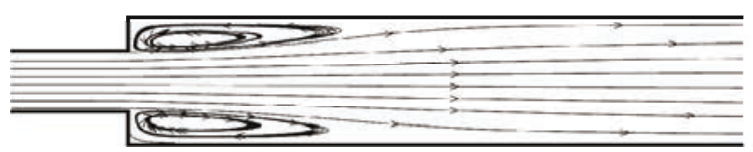

a) Before bifurcation $(R e \simeq 210)$

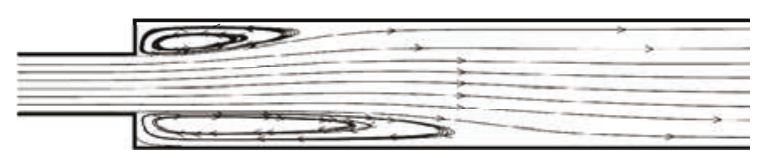

b) After bifurcation $(R e \simeq 228)$

Figure 2. Streamlines for Reynolds numbers close to the critical Reynolds number. $\left(R e_{c} \simeq 213.6\right.$ in the case of a sudden expansion $\left.E=2\right)$

Many experimental [CHE 78][FEA 90], numerical [BAT 97][DRI 97][CHI 00] or theoretical (by linear stability analysis [SHA 90], nonlinear stability analyses [MIZ 00] [RUS 99]) studies have been carried out to characterize the attachment Reynolds num-

7. On figure 2 the jet is attached to the higher wall of the sudden expansion but it can be attached randomly to one or the other of the walls. 
ber. Although the systematic dependence is not established theoretically, the critical value $R e_{c}$ decreases when the expansion ratio $E$ or the aspect ratio $A^{8}$ increases.

As Sobey and Mullin [SOB 93] have noted that the critical Reynolds number $R e_{c}$ is sensitive to the modelling parameters and to the discretization scheme, the influence of these various parameters is preliminary tested.

\subsection{Influence of the various geometrical parameters}

The influence of the grid on the phenomenon of attachment of the jet to the wall is tested for a sudden expansion with a ratio $E$ equal to 2 . Various grids have a regular step $(\Delta / h)$, identical in the vertical and the horizontal direction. It is noted that from a step $\Delta / h$ equal to 0.125 the grid size does not influence the critical Reynolds number. In what follows, for expansion ratios $E$ lower or equal to 6 , the step in the vertical direction is taken constant and equal to $\Delta / h=0.125$. On the other hand for higher ratios, in order to decrease the number of nodes, the grid in $y$ is relaxed from $y / h>6$ and $y / h<-6$. The horizontal step is always equal to $\Delta / h=0.125$. We also noted that when the exit length is greater than $L / h=40$, it does not have any influence on the Reynolds number of attachment. Thus, in order to avoid the numerical difficulties, due to the exit boundary condition $\sigma \cdot n=0$, the exit length is taken equal to $L / h=50$. Finally, we noticed that if the entry length $L_{e}$ is greater than $2 h$ the flow is not influenced. Thus, in order to have a fully developped flow, the length of the inlet is taken equal to $L_{e}=4 h$. To give an example of the number of unknowns of the computational tests, for $E=2$ the mesh consists of 2432 cells and 10169 nodes.

\subsection{Influence of the various parameters of the ANM on $R e_{c}$}

The influence of the parameter $\varepsilon$, which appears in the equation (14), and the truncation order of the series on the critical value of bifurcation is investigated. Initially the truncation order is fixed and equal to 15 , the influence of $\varepsilon$ is tested. As shown on figure 3, for $\varepsilon=10^{-3}$ the flow is symmetric ( $v$ is null) for all the Reynolds numbers. For $\varepsilon \leq 10^{-4}$, a Reynolds number exists for which the jet becomes attached to one or one of the two walls according to the choice of $\varepsilon$. The modification of this parameter introduces numerical disturbances, directing the solutions towards one of the two bifurcated branchs. The larger the parameter $\varepsilon$ is, the higher the length of each step of continuation is. Thus, for $\varepsilon=10^{-3}$ it is possible that the bifurcation is jumped. Moreover, figure 3 shows that the bifurcation is supercritical. We also noted that for $\varepsilon \leq 10^{-4}$, the critical Reynolds number $R e_{c}$ is not influenced by the value of $\varepsilon$.

Secondly, $\varepsilon$ was fixed at $10^{-8}$ and the truncation order of the series varies. We showed that the truncation order (bounded by 6 and 18) does not influence the critical

8. In the three-dimensional case the aspect ratio $A$ is defined as being the relationship between the depth of the sudden expansion $d$ and the height of the inlet channel $h(A=d / h)$. 
Reynolds number $R e_{c}$. In the following, $\varepsilon$ is equal to $10^{-8}$ and the truncation order is 15.

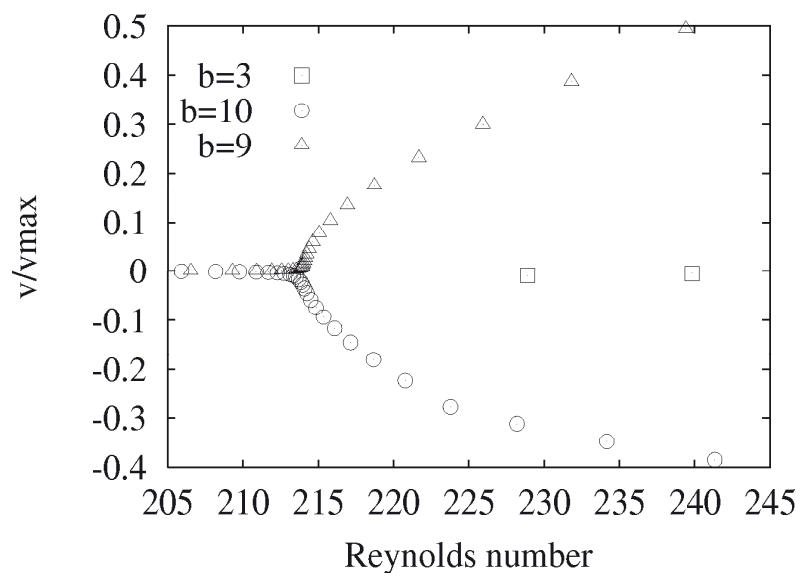

Figure 3. Evolution of vertical velocity with the parameter $\varepsilon=10^{-b}$ for a point located on the symmetry axis $(x / h=10, y / h=0)$ for $E=2$

\subsection{Evolution of $R e_{c}$ versus the expansion ratio $E$}

The numerical study is carried out for expansion ratios $E$ ranging from 1.5 to 20 . Although to our knowledge, no results exist on the limits of existence of the attachment phenomenon according to the expansion ratio, it is reasonable to think that when the walls are too distant, they do not have any influence and consequently the attachment phenomenon does not occur. Thus the study is limited to expansion ratios $E \leq 20$. For expansion ratios $1.5 \leq E<1.8$, the flow becomes attached for large Reynolds numbers?.

Table 1 gives the values obtained by the ANM and those found by other authors for $2 \leq E \leq 10$. Critical Reynolds numbers obtained are very close to those existing in the literature. These results confirm the validity of the ANM to get the critical Reynolds numbers, in the case of the sudden expansion. Confident in the validity of our approach, the study is extended to various expansion ratios which are not yet treated in the literature. From the ANM results, a law of evolution of the critical Reynolds number $R e_{c}$ according to the expansion ratio $E$ is also obtained. This relation is written for $1.8 \leq E \leq 20$ :

$$
R e_{c}=\exp \left[\beta_{1}\{\ln E\}^{\rho_{1}}\right] \quad \text { with } \beta_{1}=4.6128 \text { and } \rho_{1}=-0.4187
$$

Figure 4 shows the good agreement between the results obtained numerically by ANM and the curve of equation (17). This equation predicts the numerical results with a maximum error of $7 \%$. Therefore this equation is a fast and efficient way to estimate the critical Reynolds number.

9. $E=1.6 \rightarrow 609.3$ and $E=1.5 \rightarrow 927$ 


\begin{tabular}{|c|c|c|c|c|}
\hline Authors & $E$ & Obtention & $R e_{c}$ & $\mathrm{ANM} R e_{c}$ \\
\hline Drikakis [DRI 97] & 2 & numerical & 216 & 213.6 \\
Shapira et al. [SHA 90] & 2 & numerical & 215 & $/ /$ \\
Chedron et al. [CHE 78] & 2 & experimental & $\geq 185$ & $/ /$ \\
Battaglia et al. [BAT 97] & 3 & numerical & 80.7 & 80.5 \\
Fearn et al. [FEA 90] & 3 & numerical & 80.9 & $/ /$ \\
Shapira et al. [SHA 90] & 3 & theoretical & 82.6 & $/ /$ \\
Drikakis [DRI 97] & 4 & numerical & $53 \leq R e_{c} \leq 55$ & 53.8 \\
Drikakis [DRI 97] & 6 & $/ /$ & $33 \leq R e_{c} \leq 37$ & 35 \\
Drikakis [DRI 97] & 8 & $/ /$ & 28 & 29 \\
Drikakis [DRI 97] & 10 & $/ /$ & 26 & 25 \\
\hline
\end{tabular}

Table 1. Comparison of critical Reynolds numbers obtained by ANM to those obtained by other authors

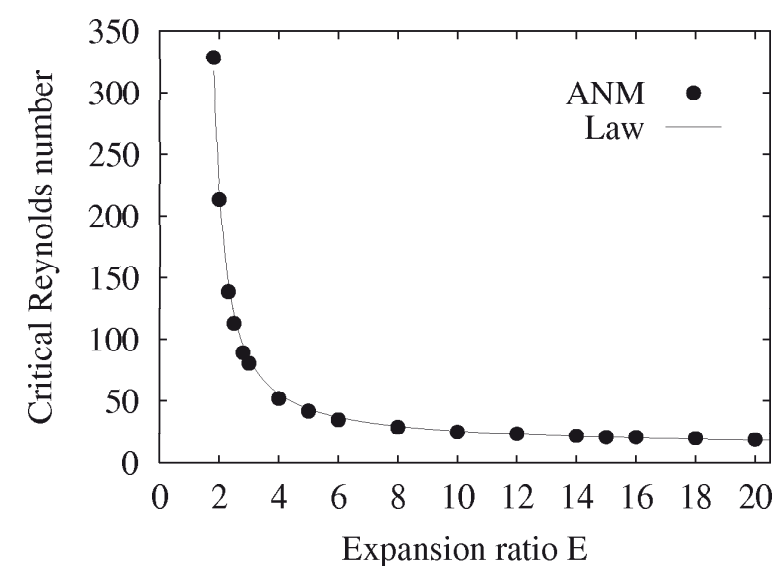

Figure 4. Variation of the attachment critical Reynolds number $R e_{c}$ according to the expansion ratio $E$

These results indicate that the critical Reynolds number $R e_{c}$ decreases when the expansion ratio of $E$ increases. For $1.8 \leq E \leq 10$ the critical Reynolds number decreases strongly varying from 329 for $E=1.8$ to 25 for $E=10$. Beyond $E=10$ the decrease is slower. In these cases the walls play a significant stabilizing role. When the walls are close to each other, a great disturbance is necessary in such a way that the jet becomes attached to one of the walls. This is explained by the fact that the recirculation zones at the inlet of the expansion are small because they do not have enough space to develop. Then a significant velocity is necessary to develop these recirculation zones and then break the flow symmetry. When the walls are distant enough, the jet is less stable and a small disturbance is needed to create the instability. In this case the recirculation zones are large at low velocity and quickly break the symmetry of the flow. 


\section{Study of a sudden expansion with divergent at the inlet}

In the configuration flow of the sudden expansion with divergent, in addition to the expansion ratio $E=h_{1} / h$ two additional geometrical parameters are introduced : the angle $\alpha$ indicating the wall divergent inclination and the ratio $E_{d}=h_{2} / h$ where $h_{2}$ is the height of the divergent exit. The addition of a short divergent, at the entry of the expansion, (figure $1 \mathrm{~b}$ )) does not change the bifurcation phenomenon. There is always a critical Reynolds number $R e_{c}$ beyond which the flow becomes asymmetrical and attached to one of the two walls of the sudden expansion. If $h_{1}=h_{2}$ (i.e $E=E_{d}$ ) Shapira et al. [SHA 90] have noted that the critical Reynolds number $R e_{c}$ is sensitive to the angle of inclination of the divergent walls. It decreases when the inclination angle increases. The recirculation zones appear on the walls of the diffuser (figure 5). As shown in figure 6 , if $h_{1}$ is not equal to $h_{2}$, the recirculation zones are located in the exit channel and not in the diffuser. Therefore, the jet is attached to one of the walls of the exit channel but not to the walls of the diffuser.

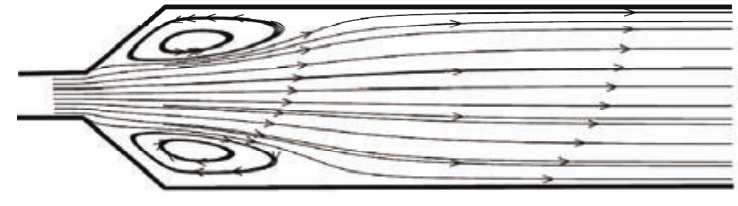

a) Before bifurcation

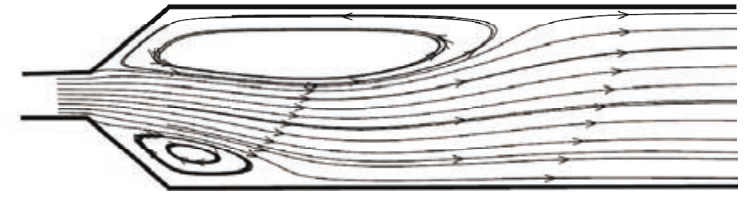

b) After bifurcation

Figure 5. Streamlines before bifurcation for $R e=53$ and after bifurcation for $R e=90$ $\left(\alpha=40^{\circ}, E=E_{d}=4\right)$

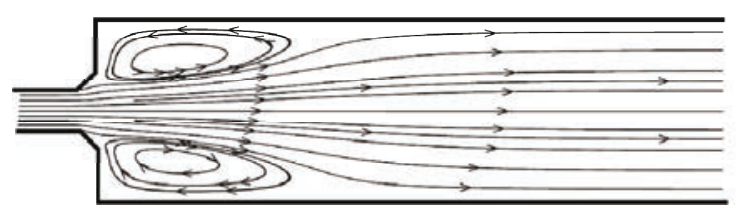

a) Before bifurcation

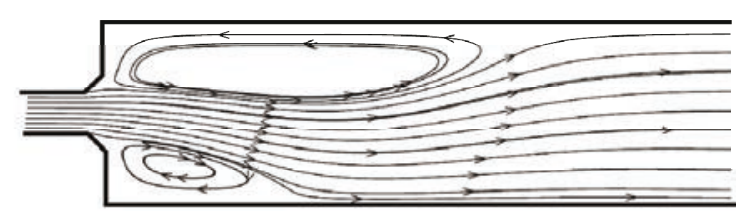

b) After bifurcation

Figure 6. Streamlines before bifurcation for $R e=51$ and after bifurcation for $R e=68$ $\left(\alpha=50^{\circ}, E=4\right.$ and $\left.E_{d}=2\right)$

In the next two parts, the influence of various geometrical parameters ( $\alpha$ and $E_{d}$ ) on the critical Reynolds number for sudden expansions with divergent is analyzed. The ratio $E_{d}$ is small and not equal to $E$ (i.e. $h_{1} \neq h_{2}$ ). 
In this section $E_{d}=h_{2} / h$ is given and the angle of the divergent is varied. If the angle $\alpha$ changes, so does the divergent length ${ }^{10} L_{d}: L_{d}$ increases when the angle $\alpha$ decreases. Figure 7 a) shows that the critical Reynolds number $R e_{c}$ decreases when the diffuser angle increases. For small angles $<50^{\circ}$ the reduction is significant. When the angle is small, the divergent walls are close to each other and their length are more significant. This increases the containment and delays the development of a perturbation. For higher angles, $R e_{c}$ decreases more slowly and tends towards the breaking value of the expansion without divergent. In this case the divergent is very short and its influence is limited.

The results obtained by ANM show that the critical Reynolds number $R e_{c}$, for a given $E_{d}$, follows the law of evolution bellow with respect to the inclination angle $\alpha$ :

$$
R e_{c}=\exp \left[a(E)\{\ln \alpha\}^{b(E)}\right]
$$

The coefficients $a$ and $b$ depend on the expansion ratio $E$. The maximum error between the results obtained by the ANM and those obtained by the evolution law (18) is $5 \%$.

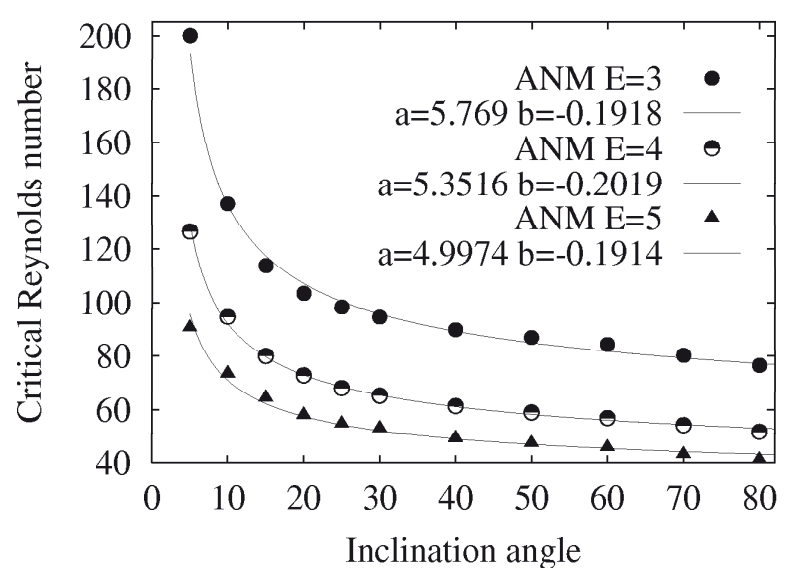

a) $E_{d}=h_{2} / h=2$ and $\alpha$ varies

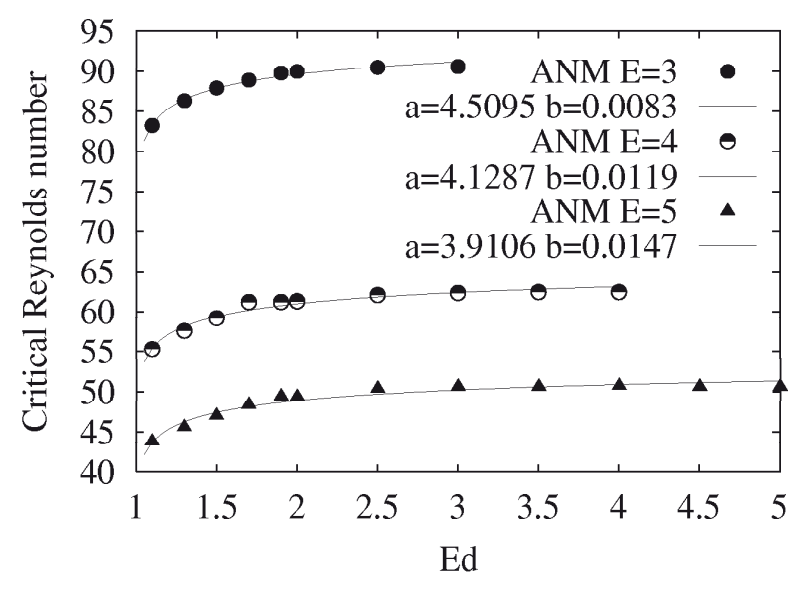

b) $\alpha=40^{\circ}$ and $E_{d}=h_{2} / h$ varies

Figure 7. Variation of the critical Reynolds number versus the parameters $E_{d}=h_{2} / h$ and $\alpha$ for various expansion ratios $E=3,4,5$

\subsection{Influence of $E_{d}=h_{2} / h$ for a given angle}

In this part $\alpha$, is fixed at $40^{\circ}$ and the ratio $E_{d}$ varies. The divergent length $L_{d}$ increases linearly with the expansion ratio $E_{d}$. For the case $E=E_{d}$, critical Reynolds numbers are similar to those obtained by Shapira et al. [SHA 90]. Figure 7 b) shows that the critical Reynolds number $R e_{c}$ increases slightly when the expansion ratio of the divergent $E_{d}$ grows. The case with $E_{d}=1$ corresponds to the sudden expansion.

10. $L_{d} / h=\left(E_{d}-1\right) / 2 \sin (\alpha)$ 
When the angle $\alpha$ is fixed, the critical Reynolds number obtained by ANM varies with the divergent expansion ratio $E_{d}$ in the following way :

$$
R e_{c}=\exp \left[a(E)\left\{\ln E_{d}\right\}^{b(E)}\right]
$$

The two functions $a$ and $b$ depend on the expansion ratio $E$. The maximum error between the results obtained by the ANM and the law of evolution (19) is $1.6 \%$.

The presence of the divergent delays the appearance of the stationary bifurcation. The longer the diffuser is, and the more significant the confinement is (i.e. the smaller $\alpha$ is), the more the loss of symmetry occurs for larger Reynolds numbers. When the diffuser is very short, it does not have almost any influence on the critical Reynolds number $R e_{c}$ and similar results to those obtained in the case of an expansion without divergent are retrieved.

\section{Open cavity}

As in the case of a sudden expansion, for small Reynolds numbers, the flow through an open cavity (figure $1 \mathrm{c}$ )) is symmetrical and is characterized by the presence of two recirculation zones of same size inside the cavity. When the Reynolds number increases, the flow becomes asymmetrical. As shown in figure 8 , if the length $L$ is small the jet becomes attached to a wall of the exit channel. If the length $L$ is large enough the jet can be attached to the walls of the cavity (figure 9). This configuration was studied experimentally and numerically by Maurel [MAU 94], Maurel et al. [MAU 96], Bouchet [BOU 96] and Mizushima et al. [MIZ 97] .

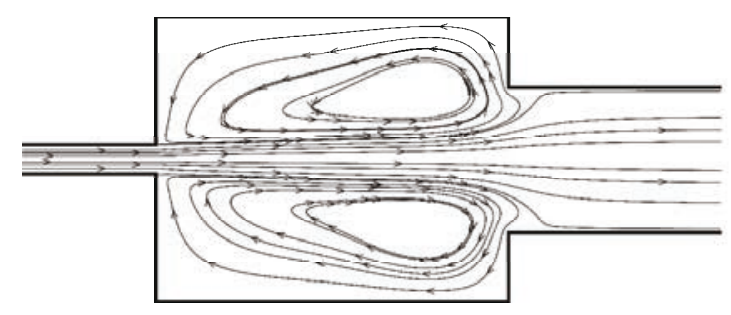

a) Before bifurcation

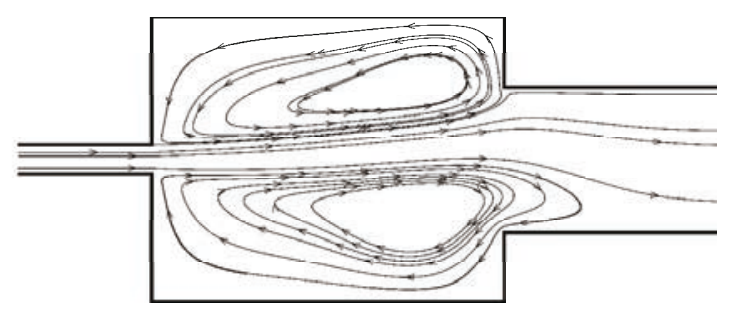

b) After bifurcation

Figure 8. Streamlines before bifurcation for $R e=58$ and after bifurcation for $R e=66$ $(L / h=12.5, W / h=10$ and $h s / h=5)$

In this section, the influence of the various geometrical parameters on the stationary bifurcation, computed with the ANM is studied. Preliminary calculations were carried out to determine a grid which does not influence the stationary bifurcation. Thus, in this section, simulations were carried out on a grid with a constant step $\Delta x / h=\Delta y / h=0.125$. The length of the exit channel is $l s=10 h s$ and the length of the inlet channel is $l e=6 h$. For example, for a square cavity $L / h=W / h=15$ and $h s / h=5$, the mesh consists of 23561 cells and 5760 nodes. 


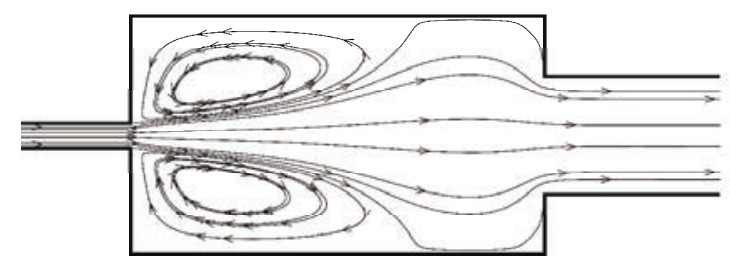

a) Before bifurcation

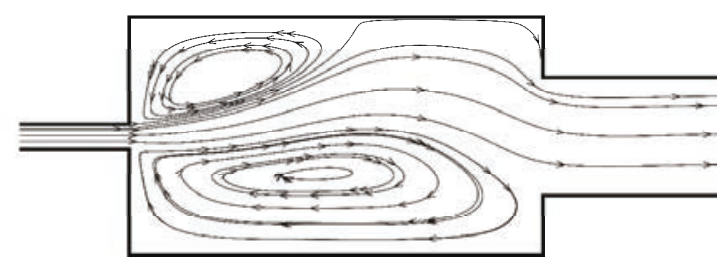

b) After bifurcation

Figure 9. Streamlines before bifurcation for $R e=23$ and after bifurcation for $R e=30$ $(L / h=17.5, W / h=10$ and $h s / h=5)$

\subsection{Influence of the cavity length $L$}

As shown in table 2, the critical Reynolds numbers $R e_{c}$ for which the jet is attached to the exit channel, obtained by the ANM, are very close to those obtained by Bouchet $^{11}$ [BOU 96]. This confirms the good validity of the code. For $W / h=25$, the jet is always attached to the exit channel for lengths $L / h \leq 22.5$. For $W / h=15$ (resp. $W / h=10$ ) the jet is attached to one wall of the exit channel for a length $L / h \leq 21.25$ (resp. $L / h \leq 13.75$ ) and to one of the walls of the cavity for higher lengths. When the distance of the horizontal walls of the cavity are large, the length of the cavity must be important to attract the jet towards one of these walls. This change of attachment walls results in a discontinuity on figure 10 a). For low lengths $L / h \leq 5$, whatever the height of the cavity is, the jet behaves as if there were no cavity and the attachement occurs for the same Reynolds number as for a sudden expansion of ratio 5 . Up to $L / h=8.75$, the height of the cavity does not influence the critical value $R e_{c}$. Beyond this length, its role becomes dominating : the larger its height is, the more important the number $R e_{c}$ is. Finally when the jet becomes attached to a cavity wall, the critical Reynolds number $R e_{c}$ is similar with that one obtained in the case of an sudden expansion with an expansion ratio $E=W / h$. In this case the exit channel does not have almost any influence on the phenomenon of attachment.

\begin{tabular}{|c|c|c|c|}
\hline$L / h$ & $\begin{array}{c}R e_{c} \text { ANM } \\
\text { based on } U_{0}\end{array}$ & $\begin{array}{c}R e_{c} \text { ANM } \\
\text { based on } U_{\text {moy }}\end{array}$ & $\begin{array}{c}R e_{c} \text { Bouchet[BOU 96] } \\
\text { based on } U_{\text {moy }}\end{array}$ \\
\hline 12.5 & 69.4 & 46.3 & $46 \pm 0.5$ \\
\hline 15 & 79.5 & 53 & $54 \pm 0.5$ \\
\hline 17.5 & 90.1 & 60 & $62 \pm 0.5$ \\
\hline 20 & 100.7 & 67.1 & $67 \pm 0.7$ \\
\hline 22.5 & 107.9 & 71.9 & $72 \pm 0.7$ \\
\hline
\end{tabular}

Table 2. Comparison between the Reynolds numbers of bifurcation obtained with the ANM and those obtained by Bouchet $(W / h=25$ and $h s / h=5)$

11. The Reynolds numbers given by Bouchet are based on mean velocity $U_{\text {moy }}$. In the case of a parabolic profile $U_{0}=3 U_{\text {moy }} / 2$ where $U_{0}$ is the highest velocity. 


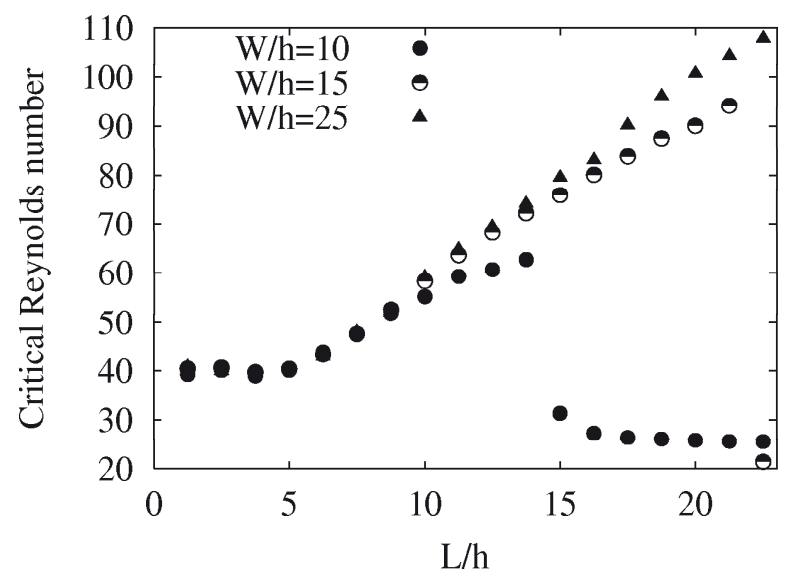

a) $h s / h=5, W / h$ fixed, $L / h$ varies

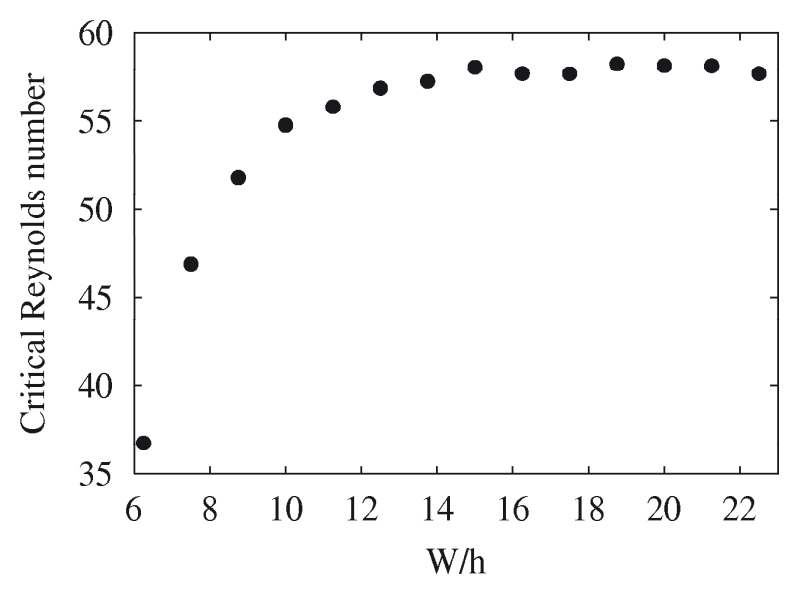

b) $h s / h=6, L / h=12.5, W / h$ varies

Figure 10. Variation of the attachment Reynolds number according to the length $L$ and the height $W$

\subsection{Influence of the cavity height $W$}

The length of the cavity and the diameter of exit are fixed $(L / h=12.5$ and $h s / h=6)$. The effect of the height on the critical Reynolds number $R e_{c}$ is tested. As shown in figure $10 \mathrm{~b}$ ), for $6.5 \leq W / h \leq 14, R_{c}$ increases strongly with the distance of the cavity walls. The more the walls are close to each other, the less the jet is stable. For higher values of $W / h$, the contribution of the horizontal cavity walls becomes less important and the critical Reynolds number $R e_{c}$ does not change.

\subsection{Influence of the exit diameter $h s$}

In this section, the length and the height of the cavity are fixed and the exit diameter $h s$ varies. The curves (figure $11 \mathrm{a}$ )) are similar to to the curves obtained in the case of a sudden expansion where the ratio expansion $E$ varies (4). On figure 11 a), $h s / h$ corresponds to the expansion ratio $E$. Thus, one can note that when the diameter of the exit channel increases, the critical value $R e_{c}$ decreases and tends towards a limit value, starting from $h s / h=8$. For small dimensions of the cavity, the results are close to those obtained in the case of a simple sudden expansion and consequently the critical Reynolds number is small.

\subsection{Square open cavity}

Finally the evolution of the critical Reynolds number in the case of a square cavity ( $W / h=L / h$ ) is studied. Figure $11 \mathrm{~b}$ ) shows that the evolution of the critical Reynolds number $R e_{c}$ is linear with respect to the cavity length. This figure confirms that when $h s / h$ decreases, the bifurcation is delayed. The evolution laws drawn on figure $11 \mathrm{~b}$ ) give a maximum error of $4.8 \%$. 


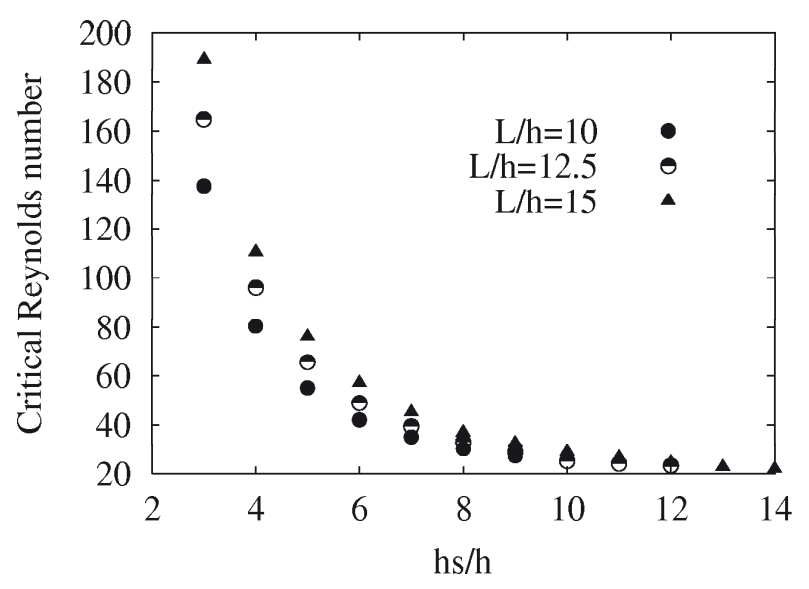

a) $L / h$ fixe and $h s / h$ varies

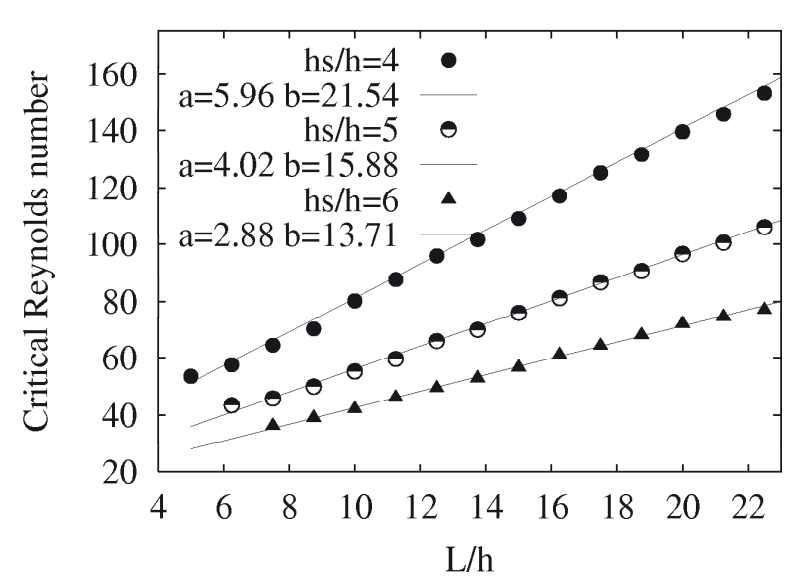

b) $h s / h$ fixe and $L / h$ varies

Figure 11. Variation of attachment Reynolds number, for a square open cavity, with the length $L / h$ and the height of the exit channel $h s / h$

To sum up, the flow through an open cavity is symmetrical for small Reynolds numbers, and becomes asymmetrical by Coanda effect. When the cavity length is small, the jet is identical to the one with no cavity and a phenomenon of attachment in the exit channel occurs. The Reynolds numbers at bifurcation tend towards those observed in a sudden expansion with an expansion ratio $E=h s / h$. When the length of the cavity $L$ is large, the jet becomes attached to the cavity walls, and the Reynolds numbers correspond to expansion ratios $E=W / h$.

\section{Conclusion}

In this article, the efficiency of the stationary ANM to detect the attachment Reynolds numbers of a jet to a wall by Coanda effect and for several geometrical configurations (sudden expansion with or without divergent and open cavity) is shown. This method allows to find similar results to those existing in the literature and to establish laws of evolution of the critical Reynolds number versus the various geometrical parameters.

For other flow configurations, like flow through a long diffuser, the Coanda effect is associated with a phenomenon of hysteresis. The dynamics of this flow, which is of interest since it is at the origin of an important sound reduction for the flow of air crossing two diaphragms in tandem inside a cylindrical pipe, cannot be obtained by resolution of the stationary Navier-Stokes equations [ALL 02]. The first computations carried out with the non stationary ANM [COC 00][JAM 02] are very encouraging and a characterization of the attachment phenomenon of this flow can be expected in a very short terms. 


\section{Bibliography}

[ABB 62] Аввотт D., KLINE S., « Experimental investigation of subsonic turbulent flow over single and double backward facing steps », Journal of basic engineering, vol. september, 1962, p. 317-325.

[ALL 02] Allery C., « Contribution à l'identification des bifurcations et à l'étude des écoulements fluides par des systèmes dynamiques d'ordre faible (POD) », PhD thesis, Université de Poitiers, France, 2002.

[BAT 90] BATOZ J. L., DhatT G., Modélisation des structures par éléments finis volume 1, solides élastiques, Hermes, France, 1990.

[BAT 97] Battaglia F., Taverner S. J., Kulkarni A. K., Merkle C. L., « Bifurcation of a low Reynolds number flows in symmetric channels », AIAA, vol. 35(1), 1997, p. 99105.

[BOU 94] Boutyour E. H., « Méthode asymptotique numérique pour le calcul de bifurcations : Applications aux struxtures élastiques », PhD thesis, Université de Metz, France, 1994.

[BOU 96] Bouchet G., « Etude expérimentale et numérique des auto-oscillations d'un jet confiné », PhD thesis, Université de Paris 6, France, 1996.

[CAD 97] CADOU J., « Méthode Asymptotique Numérique pour le calcul des branches solutions et des instabilités dans les fluides et pour les problèmes d'interaction fluide-structure », $\mathrm{PhD}$ thesis, Université de Metz, France, 1997.

[CAD 01] Cadou J., Potier-Ferry M., Cochelin B., Damil N., « An ANM for stationary Navier-Stokes equations and with Petrov-Galerkin Formulation », International Journal for numerical Methods in Engineering, vol. 50(4), 2001, p. 825-845.

[CHE 78] Chedron W., Durst F., Whitelaw J. H., « Asymmetric flows and instabilities in symmetric ducts with sudden expansion », J. Fluid Mech., vol. 84, 1978, p. 13-31.

[CHI 00] Chiang T. P., Sheu T. W. H., Wang S. K., « Side wall effects on the structure of laminar flow over a plane symmetric sudden expansion », Computers and Fluids, vol. 29(5), 2000, p. 467-492.

[COC 94] Cochelin B., « A path following technique via an asymptotic-numerical method », Computers and structures, vol. 53(5), 1994, p. 1181-1192.

[COC 00] COChelin B., Compain C., « An asymptotic numerical method for nonlinear transient analysis », Revue Européenne des Elements Finis, vol. 9, 2000, p. 113-128.

[DRI 97] DRIKAKIS D., « Bifurcation phenomena in incompressible sudden expansion flows », Physics of fluids(1994), vol. 9(1), 1997, p. 76-87.

[FEA 90] Fearn R. M., Mullin T., Cliffe K. A., « Nonlinear flow phenomena in a symmetric sudden expansion », J. Fluid Mech., vol. 211, 1990, p. 595-608.

[GUE 01] Guerin S., Allery C., Sakout. A., « Hystérésis et réduction de sons autoentretenus commandées par effet Coanda », Comptes Rendus de l'Académie des Sciences, Série 2b, vol. 329(8), 2001, p. 579-584.

[HAD 97] HADJI S., DhatT G., « Asymptotic-Newton method for solving incompressible flows », International Journal for numerical methods in fluids, vol. 25(8), 1997, p. 861878. 
[JAM 02] JAmal M., Braikat B., Boutmir S., DAmil N., Potier-FerRy M., « A high order implicit algorithm for solving nonlinear problems », Computational Mechanics, vol. 28, 2002, p. 375-380.

[KAW 76] Kawahara M., Yoshimura N., Nakagawa K., Ohsaka H., « Steady and unsteady finite element analysis of incompressible viscous fluid », International Journal for Numerical Methods in Engineering, vol. 10, 1976, p. 437-456.

[MAU 94] MaUrel A., « Instabilité d'un jet confiné », $\mathrm{PhD}$ thesis, Université de Paris 6, France, 1994.

[MAU 96] MaUrel A., ERn O., Zielinska J., Wesfreid J., « Experimental study of selsustained oscillations in a confined jet $\gg$, Physical Review E, vol. 54(4), 1996, p. 36433651.

[MIZ 97] Mizushima J., OKamoto H., Yamaguchi H., « Stability of flow in a channel with a suddenly expanded part », Physics Fluids, vol. 8(11), 1997, p. 2933-2942.

[MIZ 00] Mizushima J., Shiotani Y., « Structural instability of the bifurcation diagram for two-dimensional flow in a channel with a sudden expansion », J. Fluid. Mech., vol. 420, 2000, p. 131-145.

[POT 97] Potier-Ferry M., Damil N., Braikat B., Descamps J., Cadou J., CaO H. L., ElHaGe-Hussein A., « Traitement des fortes non-linéarités par la méthode asymptotique numérique », Comptes Rendus de l'Académie des Sciences, Série II b, vol. 324, 1997, p. 171-177.

[RUS 99] RUSAK Z., HAWA T., «A weakly nonlinear analysis of the dynamics of a viscous flow in a symmetric channel with a sudden expansion », Physics of fluids(1994), vol. 11(12), 1999, p. 3629-3636.

[SHA 90] ShapIRA M., DEGANi D., Wheis D., « Stability and existence of multiple solutions for viscous flow in suddenly enlarged channels », Computers and Fluids, vol. 18(3), 1990, p. 239-258.

[SOB 93] Sobey I., Mullin T., « Calculation of multiple solutions for the dimensional Navier-Stokes equations », Proceedings of numerical Methods in Fluid Dynamics, vol. 4, 1993, p. 417-430.

[THO 68] ThOMPSON J., WALKER A.C. H., « The non-linear perturbation analysis of discrete structural systems », International Journal of Solids and Structures, vol. 4, 1968, p. 757-768.

[TRI 96] Tri A., Cochelin B., Potier Ferry M., « Résolution des équations de Navier-Stokes et détection des bifurcations stationnaires par une Méthode Asymptotique Numérique », Revue Européenne des Eléménts Finis, vol. 5(4), 1996, p. 415-442.

[VAN 98] VAnnucci P., Cochelin B., Damil N., Potier-Ferry M., « An AsymptoticNumerical Method to compute bifurcating branches », International Journal for Numerical Methods in Engineering, vol. 41, 1998, p. 1365-1389. 